\title{
Medium Cut-Off Membranes: Incremental or Quantum Leap Innovation in Haemodialysis?
}

\author{
Mario Cozzolino ${ }^{a}$ Claudio Ronco ${ }^{b, c}$ \\ aDivision of Nephrology and Dialysis, Department of Health Sciences, University of Milan, ASST Santi Paolo e Carlo, \\ Milan, Italy; ${ }^{b}$ Department of Medicine, University of Padua, Padua, Italy; ${ }^{\mathrm{C} D e p a r t m e n t ~ o f ~ N e p h r o l o g y, ~ D i a l y s i s ~ a n d ~}$ \\ Kidney Transplant, International Renal Research Institute, San Bortolo Hospital, Vicenza, Italy
}

Native kidneys have several functions: maintain haemodynamic balance, blood pressure, electrolyte and solute concentration; excrete metabolites and liver-conjugated molecules; produce active vitamin $\mathrm{D}$; and secrete erythropoietin. According to K/DOQI and KDIGO consensus guidelines, CKD is defined as a heterogeneous group of disorders characterized by alterations in kidney structure and function, which manifest in various ways depending upon the underlying causes and the severity of disease. All of these disorders follow a progressive course and eventually lead to such a decrement in kidney function that renal replacement therapy has to be instituted. Patients with advanced CKD represent a heavy burden for the national health systems not only in terms of their diseases per se but also, and especially, because of the high rate of comorbidities and the increased risk of mortality, particularly for cardiovascular complications [1].

Haemodialysis is the form of renal replacement treatment in which fluids and solutes are removed or added to the patient's blood via an extracorporeal mechanism. When renal function declines, the capability of the kidney to remove metabolites decreases accordingly; these metabolites are defined as uraemia retention molecules (uraemic toxins), and the main aim of dialysis is to clear them as much as possible, in order to protect the body from their potential toxic effects, trying to reproduce native kidney functions.

Most of these toxins, due to their molecular weight (MW) and/or binding to proteins, are not removed by dialysis, and thus they represent a crucial therapeutic challenge. Accumulation of uraemic compounds impacts on the functions of cells which are involved in myocardial and vascular functions, such as leukocytes, endothelial cells, vascular smooth muscle cells and platelets, with alterations in infection response and promotion of chronic inflammation, oxidative stress, apoptosis, fibrosis, endothelial dysfunction, and deficiency of endogenous calcification inhibitors. Eventually, these abnormalities lead to cardiovascular and cerebrovascular events, alteration of mineral bone metabolism, and disease progression up to ESRD. The result is an increase in all-cause mortality rate, especially cardiovascular disease-dependent deaths [2].

In particular, middle uraemia retention molecules include compounds with a MW ranging between 500 and $58,000 \mathrm{Da}$, with most molecules having a $\mathrm{MW}>10 \mathrm{kDa}$. The vast majority of middle molecules is represented by proteins derived from endogenous metabolism (either karger@karger.com

(c) 2020 S. Karger AG, Basel

www.karger.com/bpu

Karger!
Mario Cozzolino

Renal Division and Laboratory of Experimental Nephrology, Dipartimento di Scienze della Salute, Università di Milano, ASST Santi Paolo e Carlo Hospital Via A. di Rudinì, 8, IT-20142 Milan (Italy)

mario.cozzolino@unimi.it 
primary products or their metabolites) which are cleared less and/or synthesized in response to uraemic environment changes. Toxicity affects mainly the cardiovascular system, pro-fibrotic pathways and inflammation [3]. Furthermore, most of the compounds belonging to proteinbound uraemic toxins (PBUTs) are small and derive from gastrointestinal metabolism. Due to protein binding, these solutes cannot be removed by dialysis; only for a few of them, specific therapeutic strategies have been implemented (i.e., folic acid supplementation for homocysteine). They mostly impact on inflammation and on the cardiovascular system. Therefore, research is now concentrating on the development of therapeutic strategies aimed at reducing toxin production (i.e., gut microbiome as a potential new target) and of dialysis techniques able to remove middle and protein-bound molecules [4].

Interestingly, high-flux haemodialysis (HF-HD) is defined by its capability to more effectively remove medium-to-large uraemic toxins $(\mathrm{MW}>1,000 \mathrm{Da})$; furthermore, it offers a high biocompatibility due to the use of ultrapure membranes [5]. Clinical trials have shown that HF-HD improves prognosis and cardiovascular outcomes with respect to low-flux membranes, especially in high-risk patients. However, no clear clinical impact has been found in terms of long-term treatment, and HF-HD and online haemodiafiltration are still inadequate in terms of middle molecule clearance, even if they are surely better than conventional LF-HD.

Hemodiafiltration is a high-flux-requiring technique which combines solute and fluid removal by diffusion and convection. HDF is capable of removing more fluid than necessary, and thus reinfusion is needed; unfortunately, reinfusion fluid has to be extremely pure and apyrogenic; hence, this technique is very expensive. Online haemodiafiltration is a variant which allows to use purified dialysate as an infusion fluid; in this way, up to 25-30 $\mathrm{L}$ of reinfusion fluid can be obtained without a high cost. Many studies comparing HF-HD and HDF gave discordant results in terms of mortality rates because of the high burden of comorbidities ESRD patients have [6]. To summarize, HDF appears to have a better cardioprotective action and to show an improvement in immune functions with respect to conventional LF-HD, due to the more efficient convective transport of medium MW and PBUTs; this result can be obtained thanks to high convective volume clearances achieved with HDF. Unfortunately, it is very hard for patients to actually reach the desired convective clearance in everyday clinical practice (at least $21-$ $23 \mathrm{~L}$ of convective volume per session). Hence, it is of major importance to implement a diffusive technique
Table 1. Middle-MW uraemic toxins

MW >500 Da; medium-/high-cut-off membranes

AGES and AOPP

Adrenomedullin

Atrial natriuretic peptide

$\beta 2$-Microglobulin

$\beta$-Endorphin

Cholecystokinin

Clara cell protein

Complement factor D

Cystatin C

Degranulation-inhibiting protein I

Delta-sleep-inducing peptide

Endothelin

FGF-23

Hyaluronic acid

IL-1 $\beta$

IL-6

Kappa-Ig light chain

Lambda-Ig light chain

Leptin

Methionine-enkephalin

Neuropeptide Y

PTH

Retinol-binding protein

TNF- $\alpha$

MW, molecular weight; IL, interleukin; TNF- $\alpha$, tumour necrosis factor-alpha.

able to remove medium-MW uraemic toxins, and this can be achieved by developing dialysis membranes with better structure and permeability.

The most recent and promising advance in the field of haemodialysis is represented by the development of medium-cut-off (MCO) high-retention-onset membranes. These filters have enhanced permeability and selectivity, and very high MW retention onset and cut-off, with the latter being close to the MW of albumin (Table 1). Basically, they are permeable enough to obtain a high rate of middle-molecule clearance without allowing to lose too much albumin in the process [7].

The term expanded haemodialysis (HDx) has been introduced to describe a dialytic modality in which diffusion and convection are combined inside a hollow-fibre dialyser containing an MCO membrane. With respect to HDF (in which convective removal is determined by the volume of exchanged fluid), in HDx convection is made possible by the intrinsic features of the MCO dialyser [8]. Middle molecules with MWs of 500-2,000 Da are removed better by peritoneal dialysis compared to haemo- 


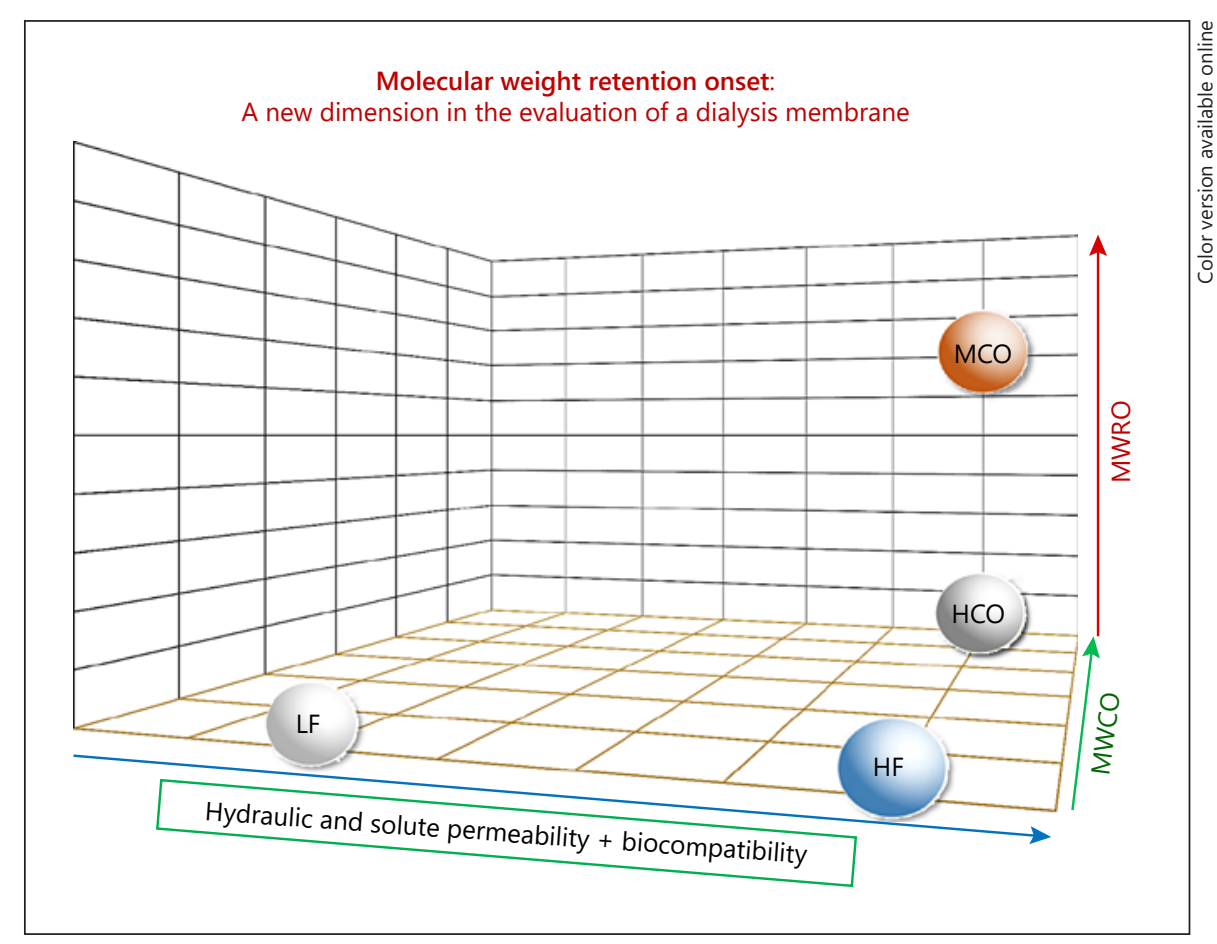

Fig. 1. Three important dimensions should be considered for the evaluation of new membranes. a: Flux (blue line). The move from cuprophan to high-flux membranes has made it possible to perform new therapies including high-flux dialysis and haemodiafiltration improving removal of middle-MW solutes. With the increase in hydraulic permeability, biocompatibility was also improved due to the synthetic biomaterial composition. b: Cut-off (MWCO) (green line). Membrane cut-off is defined by the MW at which sieving coefficient is 0.1 . The progressive increase in permeability has led to an increased pore size distribution. The possibility to remove large solutes such as myoglobin or even cytokines is guaranteed by the presence of high cut-off of the new membranes

dialysis using cuprophane membrane, improving uraemic neuropathy. These new MCO membranes have been developed to remove middle molecules.

In Figure 1, we summary the new dimension in the evaluation of dialysis membrane. From an effectiveness point of view, research is showing promising results. Studies by Kirsch et al. [9] showed how different prototypes of MCO membranes used in a single session of HDx were able to efficiently remove middle and large molecules such as $\beta_{2}$-microglobulin, free light chains (FLCs), complement factor D, myoglobin, and phosphorus, even if pre-dialysis levels were not affected; a quite important albumin loss was still seen, but patients were asymptomatic. However, some degree of albumin leak could be theoretically accepted since this would imply concomitant removal of some PBUTs. Furthermore, the uraemic envi- that, however, results in possible leakage of albumin due to pores with a dimension larger than the molecular radius of the albumin molecule. c: Retention onset (MWRO) (red line). Membrane retention onset is defined by the MW at which sieving coefficient is 0.9 . This is a new parameter that describes the innovative characteristics of MCO membranes, in which the steep sieving coefficient curve is achieved thanks to a narrow distribution of the pore size with a cut-off close to the MW of albumin. This feature guarantees maximal removal of large middle molecules with marginal or negligible losses of albumin, and it is the basis for a new therapy called HDx. MW, molecular weight; MCO, medium cut-off; HDx, expanded haemodialysis.

ronment can alter the structure of albumin, among the other proteins, via carbamylation or oxidation, leading to malfunction (in terms of hormone and drug binding and of acid-base balance) and toxicity (i.e., carbamylated albumin is associated with greater mortality rates and eritropoiesis-stimulating agents resistance). Another study by Zickler et al. [10] evaluated the capability of MCO membrane HDx to impact on the chronic inflammatory milieu: analysis of tumour necrosis factor-alpha and interleukin-6 mRNAs in circulating monocytes showed that cytokine gene expression was significantly lower after 4 weeks of HDx (mRNA levels were $82 \%$ with respect to HDF, 95\% CI 74-91\%), whereas IL-10 was significantly increased. In this study, albumin leak was $<2 \mathrm{~g} /$ session, and the patient did not develop any symptoms related to hypalbuminaemia. Moreover, serum of healthy patients 
enriched with cytokines and processed through a miniature model of dialysis, with HF-HD, HCO, and MCO membranes, showed a better clearance of interleukin- 6 and induced less vascular calcification in an in vitro culture when processed with the MCO dialyser.

The pilot study by Bushljetik et al. [11] shows that MCO membranes, compared to high-flux membranes, have greater permeability for middle molecules. In fact, a higher average removal rate for all the uraemic toxins with the Theranova dialyser was observed in 12 weeks of follow-up $(62.7 \%$ for beta- 2 microglobulin, $56.9 \%$ for myoglobin, $63.5 \%$ for FLC-k, and $54.6 \%$ for FLC- $\lambda$ ).

The introduction of MCO membranes in clinical practice is one of the most relevant innovations in the field of haemodialysis. These filters are capable of removing medium-high MW uraemic toxins without the need for high convective volumes and without a significant albumin loss [12]. Since uraemic toxins have been linked to the development of the chronic inflammatory state typical of ESRD and dialysis, to the formation of vascular calcification and, consequently, to the incidence and severity of adverse cardiovascular events, the implementation of a dialytic technique able to better clear these molecules could have a major impact on the outcomes of haemodialysis patients. One of the most promising results obtained from these studies is the decreased incidence of infectious episodes in patients treated with the new Theranova ${ }^{\circledR}$ filter. It is well known that haemodialysis patients are more subjected to infections than the general popula- tion; among the predisposing factors there are the high prevalence of diabetes, the presence of permanent vascular accesses and the secondary immunodeficiency dependent on the accumulation of uraemic toxins and the chronic inflammatory state. Thus, we can hypothesize that the reduction of infectious episodes in Theranova ${ }^{\circledR}$ dialysed subjects might depend on a decrease in the chronic inflammation. Finally, further randomized clinical trials are necessary to better evaluate the relationship between middle molecule removal by $\mathrm{MCO}$ and hard outcomes in dialysis setting.

\section{Conflict of Interest Statement}

M.C. has been a consultant or advisory board member for Baxter in the past 3 years; C.R. has been a consultant or advisory board member for ASAHI, Baxter, bioMerieux, B. Braun, CytoSorbents, Estor, Fresenius Medical Care, General Electric, Medtornic, and Toray in the past 3 years.

\section{Funding Sources}

There are no funding sources to declare.

\section{Author Contributions}

All authors contributed equally to the creation, writing, and revision of the manuscript and approved submission.

\section{References}

1 Cozzolino M, Mangano M, Stucchi A, Ciceri $\mathrm{P}$, Conte F, Galassi A. Cardiovascular disease in dialysis patients. Nephrol Dial Transplant. 2018 Oct 1;33(Suppl 3):iii28-34.

2 Cozzolino M, Galassi A, Pivari F, Ciceri P, Conte F. The cardiovascular burden in endstage renal disease. Contrib Nephrol. 2017; 191:44-57.

3 Barreto FC, Barreto DV, Canziani MEF. Uremia retention molecules and clinical outcomes. Contrib Nephrol. 2017;191:18-31.

4 Massy ZA, Liabeuf S. Middle-molecule uremic toxins and outcomes in chronic kidney disease. Contrib Nephrol. 2017;191:8-17.

5 Ronco C. Hemodiafiltration: technical and clinical issues. Blood Purif. 2015;40(Suppl 1): 2-11.
6 Blankestijn PJ, Grooteman MP, Nube MJ, Bots ML. Clinical evidence on haemodiafiltration. Nephrol Dial Transplant. 2018 Oct 1; 33(Suppl 3):iii53-8.

7 Ronco C, Reis T, Cozzolino M. Rationale for medium cutoff membranes in COVID-19 patients requiring renal replacement therapy. Nephron. 2020 Aug 4;144(11):550-4.

8 Ronco C, Marchionna N, Brendolan A, Neri M, Lorenzin A, Martínez Rueda AJ. Expanded haemodialysis: from operational mechanism to clinical results. Nephrol Dial Transplant. 2018 Oct 1;33(Suppl 3):iii41-iii47.

9 Kirsch AH, Lyko R, Nilsson LG, Beck W, Amdahl M, Lechner P, et al. Performance of hemodialysis with novel medium cut-off dialyzers. Nephrol Dial Transplant. 2017 Jan 1; 32(1):165-72.
10 Zickler D, Schindler R, Willy K, Martus P, Pawlak M, Storr M, et al. Medium cut-off (MCO) membranes reduce inflammation in chronic dialysis patients-a randomized controlled clinical trial. PLoS One. 2017 Jan 13;12(1):e0169024.

11 BPU-2020-5-50/R2 Resubmission - efficacy of medium cut-off dialyzer and comparison with standard high-flux hemodialysis. Authors: Irena Rambabova Bushljetik (Corresponding Author), Lada Trajceska (Co-author), Sefedin Biljali (Co-author), Trajan Balkanov (Co-author), Petar Dejanov (Co-author), Goce Spasovski (Co-author). Accepted in press.

12 Cozzolino M, Magagnoli L, Ciceri P, Conte F, Galassi A. Effects of a medium cut-off (Theranova) dialyser on haemodialysis patients: a prospective, cross-over study. Clin Kidney J. 2019 Nov. 